\title{
Effective factors on Auditory Brainstem Response test in Newborns
}

\author{
Mozafar Sarafraz ${ }^{1}$, Maryam Kardooni ${ }^{1}$ and Somayeh Araghi $^{2 *}$ \\ ${ }^{1}$ Associated Professor of otolaryngology, Head and neck surgery, Hearing \& Speech \\ Research Centre, Ahvaz Jundishapur University of Medical Sciences, Ahvaz, Iran. \\ ${ }^{2}$ Resident of otolaryngology, Head and neck surgery, Hearing \& Speech Research Centre, \\ Ahvaz Jundishapur University of Medical Sciences, Ahvaz, Iran.
}

DOI: http://dx.doi.org/10.13005/bbra/1935

(Received: 19 July 2015; accepted: 15 October 2015)

\begin{abstract}
Promotion of community health careis one of the major health and treatment policies. In this regard, prevention is considered the primary, most important and effective level of health care. Given the high prevalence of congenital (sensorineural) hearing loss (nearly 1/1000 to 3/1000 of live births) large numbers of neonates with congenital hearing loss will be born annually. Hearing loss will cause adverse effects on social, cognitive and speech development of these children. Therefore, early, even neonatal, diagnosis and treatment of hearing loss has been recommended in order to prevent such complications. This study has been conducted with the aim of hearing screening of the newborns and evaluating the effective factors on Auditory Brainstem Response (ABR) test. In this descriptive study all included newborns were examined by TEOAE test during first week after birth. The result of the test was interpreted as "Pass" or "Refer". In case of "Refer", the test was repeated 3 weeks later. In neonates with the test result of "refer" in the second step, the ABR was done.The following risk factors were evaluated: 1) History of NICU admission for more than 48 hours 2) History of neonatal exchange transfusion 3) Family history of hearing deficiency 4) Birth weight 5) Administration of aminoglycosides in neonatal period. Of the total5850 evaluated newborns, 5148 (88\%) gained criteria of "Pass" in first step of the test, while 702 cases (12\%) were "Referred" to the second step and $468(8 \%)$ had impaired ABRtest. Related risk factors were evaluated in neonates with the abnormal OAE test or in those with the result of "Refer". This showed that: 1) History of NICU admission for more than 48 hours was positive in $4.8 \%$ of normal newborns but in $55 \%$ of neonates with abnormal ABR test, and the difference was significant $(P<0.001) .2)$ Family history of hearing deficiency was positive in $4.4 \%$ of normal newborns but $5.9 \%$ of neonates with abnormal ABR test, so there was not a significant relation $(P=0.191)$. 3) Low birth weight $(<1500 \mathrm{gr})$ was found in $1.5 \%$ of normal newborns and $11.5 \%$ of neonates with abnormal ABR test, and the relation was statistically significant $(\mathrm{P}<0.001)$. 4) History of neonatal exchange transfusion was present in $2.8 \%$ of normal newborns but $2.1 \%$ of neonates with abnormal ABR test, so there was not a significant relation $(P=0.563) 5)$ Administration of aminoglycosides in neonatal period was present in $2 \%$ of normal newborns but $20 \%$ neonates with abnormal ABR test, which were significantly different $(\mathrm{P}<0.001)$. The results showed a significant relation between abnormal ABR test and history of NICU admission for more than 48 hours, low birth weightand administration of aminoglycosides in neonatal period. Because of high prevalence of congenital hearing loss and it's adverse effects on child development, screening of hearing sense is recommend as soon as clinically possible after birth. In addition, paying attention to the related risk factors and particular focus on neonates with such risk factors seems beneficial. Among the four risk factors evaluated in this study, abnormal ABR test was significantly related with the history of NICU admission for more than 48 hours, low birth weight ( $<1500$ gr), and administration of aminoglycosides in neonatal period. However the two risk factors of positive family history and history of neonatal exchange transfusion were not significantly related with hearing loss.
\end{abstract}

Key words: hearing screening, congenital hearing loss, Auditory Brainstem Response, neonate

\footnotetext{
* To whom all correspondence should be addressed. Fax: +986132921838;

E-mail: savesina@yahoo.com
} 
Congenital hearing loss (sensorineural) has a high reported frequency of 1 to 3 cases in 1000 live births according to American and European studies, reflecting the fact that a large number of neonates will be born annually with congenital hearing loss disorders ${ }^{1,2}$. Loss of hearing sense in these children will led to impaired speech and social-cognitive development and delayed detection might result in permanent disabilities. Investigations have emphasized on the importance and efficacy of early diagnosis and interventions leading to beneficial effects on language skills in children with hearing loss ${ }^{3,4}$.

Even an average decrease in hearing loss will have a profound impact on language and speech development, thus early detection of hearing loss is of great importance in order to determine the appropriate interventions as soon as possible. Any delay in treatment will cause permanent damages in language and speech development. Unfortunately delayed interventions do not help much to the deaf/hard of hearing children and they will experience developmental delay with lower language and speech abilities compared to normal children of the same age $\mathrm{e}^{3,4}$.

Establishment of Joint Committee on Infant Hearing (JCIH) had a significant effect on the screening process in the United States. This Committee was first established in 1969, and in 1972, with the new findings in the ability of detecting congenital hearing abnormalities using High Risk Registry (HRR)data, the committee asserted the necessity of early screening, diagnosis and follow up of the at risk children. These newborns were referred for hearing tests within the first two months of life ${ }^{5}$. In years 1982, 1990 and 1994 HRR criteria underwent some revisions. According to the 1994 version, HRR criteria includes: 1.family history of hereditary sensorineuralhearing loss 2.history of intrauterine infections (such as toxoplasma gondii, CMV, etc) 3.craniofacial anomalies 4.birth weight lower than 1500 gr 5.hyperbilirubinemia at the level that needsblood exchange 6.history of treatment with ototoxic medications 7.bacterial meningitis 8.one minute APGAR score of 4-5 or five minute APGAR score of 5-6 9.mechanical ventilation for 5 days or more 10.other signs or symptoms associated with known syndromes that include sensorineural or conductive hearing loss.
JCIH recommends using the HRR criteria to reduce the risk of misdiagnosis delayed diagnosis of neonates and infants with hearing loss so that lower proportions of them will encounter serious complications of this disability(6). A study showed that in centers with hearing loss screening facilities, $2-5 \%$ of the neonates with one or more of the mentioned risk factors had moderate to severe hearing loss. On the other hand, $50 \%$ of the children with moderate to severe congenital hearing loss had no risk factors for it. This shows that using HRR to determine the indications for evaluating hearing sense might lead to misdiagnosis and delayed intervention in newborns without any risk factors. Therefore in 1994 this committee recommended hearing screening at birth for all newborns ${ }^{6}$.

In the year 2000 in a statement -which is a milestone for all specialties related with hearing loss- it was decided that universal hearing screening must be performed for all newborns within the first three months of life using the two screening methods of TEOAE or $\mathrm{ABR}^{6}$.Using these two new technologies, auditory system function can be measured objectively in infants. Both methods are considered non-invasive modalities recording physiologic activities based on normal hearing function and can be easily used in newborns. TEOAEare very low intensity sounds that can be measured using a microphone placed in external auditory canal. These sounds arethe energy emitted by the cochleain response to the acoustic stimulation of outer hair cells (OHC) by noise that leads to amplification of basal membrane and production of the energy (signal). The function of hearing cochlea can be evaluated by measuring the ratio of signal to noise ${ }^{7}$.

\section{METHODSAND MATERIALS}

A total of 5850 newborns were screened by TEOAE test followed by ABR test. This was a descriptive study. A questionnaire was completed for each newborn that included data about family history, address, risk factors and the results of the OAE test.

A thorough physical examination was performed for each newborn, and then the auditory canal was cleaned if there were discharges or vernix and the OAE probe was fixed in the ear. A click 
with 80-85 dB was sent to cochlea. Bandwidthwas $1-5 \mathrm{kHz}$ and was emitted with a rate of 75 to 100 pulses per second. When the emission was adequate and met the "pass" criteria, the recording was stopped. When the ratio of signal to noise was equal to or higher than $6 \mathrm{~dB}$ in at least two of the four bands, the result was also considered "pass" which was checked separately in 1, 2, 3 and $4 \mathrm{kHz}$ frequencies. If the criteria were not met, the result was "refer" and the test was repeated within three weeks. If by then the result of the OAE test was again "refer", the ABR test was performed for the newborn.

Data recorded in the questionnaire included birth weight, history of NICU admission, history of blood exchange due to hyperbilirubinemia, familial history of hearing loss and aminoglycoside administration in neonatal period. Data was analyzed using SPSS software version 13 and the chi-square statistical test.

\section{RESULTS}

Of the total 5850 studied newborns, 5148 cases (88\%) gained the "pass" criteria in OAE test and 702 cases (12\%) were referred to the second step in which 468 cases (8\%) showed an impaired ABR test. All the studied newborns were evaluated for the JCIH risk factors of hearing loss that led to the following findings:

A. History of NICU admission longer than 48 hours was found in $4.8 \%$ of the normal infants and in $55 \%$ of the newborns with impaired ABR test and the difference was statistically significant $(\mathrm{P}<0.001)$.

B. A positive history of hearing loss was recorded in $4.4 \%$ of the normal cases and $5.9 \%$ of the abnormal ABR test cases and the difference was not significant $(\mathrm{P}=0.191)$.

C. Very low birth weight (VLBW: lower than 1500 gr) was seen in $1.5 \%$ of the normal infants while $11.5 \%$ of the newborns with impaired ABR test were found to be VLBW. This relation was statistically significant $(\mathrm{P}<0.001)$.

D. History of blood exchange was positive in $2.8 \%$ and $2.1 \%$ of the normal and impaired ABR test infants respectively with no significant difference $(\mathrm{P}=0.563)$.

E. History of aminoglycoside administration in neonatal period was positive in $2 \%$ of the normal cases and $20 \%$ of the ABR impaired cases. This difference was found statistically significant $(\mathrm{P}<0.001)$

\section{DISCUSSION}

The present study aimed to screen hearing loss in newborns using $\mathrm{OAE}$ and $\mathrm{ABR}$ tests and evaluate the effective factors on the test responses.

Given the high prevalence of congenital hearing loss, a large population of deaf or hard on hearing neonates is born annually and considering the significant importance of hearing ability in development of speech, language, cognition and comprehension, early detection and treatment of this disability plays a great role in health care of this population. In several studies, such as the one by Yoshinaga-Itano et al on 150 deaf and hard on hearing children and infants, it was demonstrated that speech language development of children whose hearing loss was detected at the first six months of life is significantly better(4). The first six months of life is a very critical and sensitive period in primitive speech language development. If hearing loss is detected and corrected within this period, the future disabilities can be prevented and the infant with hearing loss might reach the normal speech and language developmental level as healthy children. Otherwise, after six month of age, even with costly advanced interventions such as hearing aids or cochlear implants the child will still have developmental delay compared and rehabilitation measures including speech therapy will be required $(3,4)$.

With the advanced technologies in audiometry, the two methods of OAE and ABR are available for hearing screening before six months of age. The importance and necessity of universal screening in all newborns become more obvious when considering the high percentage of deaf and hard on hearing children in the population and the followed high costs of rehabilitation, speech therapy and other disabilities. ABR and OAE tests are perfect tools for universal screening of all newborns since they are non-invasive and cost benefit.

Meanwhile special attention must be paid to hearing loss risk factors. During years of 1982, 
1990, 1994 and 2000 the JCIH has identified risk factors of hearing loss that are mentioned elsewhere $^{5}$. Their studies showed that $2-5 \%$ of the newborns with one or more of these risk factors had moderate to severe hearing loss. In other word, the majority of newborns with e" 1 risk factors were either normal or had mild hearing loss. Thus relying on HRR criteria for screening of at risk newborns will result in misdiagnosis in a large proportion of cases with hearing loss.

In a study by Thompson, Diane and et al (2001) it is described that Among infants in a neonatal intensive care unit (NICU), the risk of moderate-to-severe PHL is 10 to 20 times higher than in the general population. In addition to NICU admission, the Joint Committee on Infant Hearing high risk guidelines specify 4 other risk factors (Neonatal intensive care unit admission for 2 or more days

2. Usher syndrome, Waardenburg syndrome, or findings associated with other syndromes known to include hearing loss

3. Family history of hereditary childhood sensorineural hearing loss

4. Congenital infections such as toxoplasmosis, bacterial meningitis, syphilis, rubella, cytomegalovirus, and herpes

5. Craniofacial anomalies, including morphologic abnormalities of the pinna and ear canal) ${ }^{8}$.

In our study NICU admission longer than 48 hours was significantly related with hearing loss which is probably due to mechanical ventilation with positive pressure, higher possibility of infection, higher possible anomalies of cardiac, gastrointestinal or auditory systems and the associated syndromes. Hearing loss was also significantly higher in newborns with birth weight lower than 1500 gr which is probably because the same factors causing fetal malnutrition and intrauterine growth retardation or LBW can have adverse effects on auditory system as well.

According to our findings, positive family history of hearing loss was not related with hearing loss of newborns. It must be considered that many of hearing disorders leading to neonatal hearing loss are not hereditary and most of our study populations were from these groups. Moreover, no relation was found between history of blood exchange and neonatal hearing loss which reflects the high quality care and early exchange in these newborns before any hearing complications were caused by severe jaundice (Kernicterus).

Hearing loss was significantly more common in neonates with positive history of aminoglycoside administration due to higher sensitivity of newborns to the ototoxicity of these medications.

\section{CONCLUSION}

A total of 5148 newborns were studied in this investigation and risk factors of hearing loss such as family history of hearing loss, history of blood exchange, NICU admission longer than 48 hours, birth weight lower than 1500 gr and aminoglycoside administration in neonatal period were evaluated. TEOAE screening test was performed in all newborns before discharge, and if the test was impaired, ABR test was performed as well. Finally, hearing loss was diagnosed in 468 (nearly $8 \%$ ) of the studied newborns. A significantly higher frequency of NICU admission longer than 48 hours, birth weight lower than 1500 gr, and aminoglycoside administration was found in this group.

\section{REFRENCES}

1. Onoda, R.M., M.F.d. Azevedo, and A.M.N.d. Santos, Triagem auditiva neonatal: ocorrência de falhas, perdas auditivas e indicadores de riscos. Brazilian Journal of Otorhinolaryngology, 2011; 77: 775-783.

2. Wood, Sally A., Graham J. Sutton, and Adrian C. Davis. "Performance and characteristics of the Newborn Hearing Screening Programme in England: The first seven years.” International Journal of Audiology 2015; 1-6.

3. Uus, K. and J. Bamford, Effectiveness of Population-Based Newborn Hearing Screening in England: Ages of Interventions and Profile of Cases. Pediatrics, 2006; 117(5): p. e887-e893.

4. Yoshinaga-Itano, C., et al., Language of Earlyand Later-identified Children With Hearing Loss. Pediatrics, 1998; 102(5): p. 1161-1171.

5. Joint Committee on Infant Hearing 1994 Position Statement. Int J Pediatr Otorhinolaryngol, 1995; 32(3): 265-74.

6. Year position statement: principles and guidelines for early hearing detection and intervention 
programs. Joint Committee on Infant Hearing, American Academy of Audiology, American Academy of Pediatrics, American SpeechLanguage-Hearing Association, and Directors of Speech and Hearing Programs in State Health and Welfare Agencies. Pediatrics, 2000; 106(4): p. 798-817.

7. John, M., A. Balraj, and M. Kurien, Neonatal screening for hearing loss: pilot study from a tertiary care centre. Indian J Otolaryngol Head Neck Surg, 2009; 61(1): p. 23-6.

8. Thompson, Diane C., Heather McPhillips, Robert L. Davis, Tracy A. Lieu, Charles J. Homer, and Mark Helfand. "Universal newborn hearing screening: summary of evidence.” Jama 2001; 286(16): 2000-2010. 